\title{
A new risk scoring system for prediction of long-term mortality in patients on maintenance hemodialysis
}

Haruki Itoh ${ }^{1 *}$, Hiroshi Kawaguchi ${ }^{2}$, Yoichiro Tabata ${ }^{3}$, Noriyoshi Murotani ${ }^{4}$, Tomoko Maeda ${ }^{5}$, Hidetaka Itoh ${ }^{6}$ and Eiichiro Kanda ${ }^{7}$

\begin{abstract}
Background: It has been reported that the survival of hemodialysis (HD) patients is poor, and the leading cause of death is cardiovascular disease. To identify high-risk patients and treat them carefully, we developed a scoring system to evaluate their 15-year prognosis in a prospective cohort study.

Methods: We analyzed data from 312 and 310 patients to develop and validate the prediction model, respectively. The association of potential risk factors with death was tested by Cox proportional-hazards analysis, and a risk scoring model was developed. Then, the model was validated.

Results: Two hundred patients (64.1 \%) in the cohort for model development died. Six independent prognostic factors were retained in the final model, and each was assigned a score proportional to its regression coefficient: 65 years or older, 3; diabetic nephropathy, 3; hypotension, 1; pre-HD cardiothoracic ratio $\geq 50 \%$, 1; pre-HD BNP $\geq 250$ pg/mL, 1; and pre-HD numbers of abnormal findings on electrocardiograms $=0,1,2$, or larger, $0,1,5$. The patients were categorized as follows with their scores: group 1 (low risk), 0; group 2, 1 to 3; group 3, 4 to 5; and group 4 (high risk), 6 and higher. In the cohort for model validation, groups 2 to 4 showed a higher risk than group 1: group 2, hazard ratio 4.66 (95 \% confidence interval 2.25, 9.64); group 3, 13.62 (6.48, 28.63); and group 4, $20.86(9.60,45.31)$.
\end{abstract}

Conclusions: A new risk scoring system for predicting 15-year mortality was developed. This system may be useful for evaluating HD patients' prognosis.

Keywords: Dialysis, Mortality, Death, Cardiovascular disease, Risk score, Diabetes mellitus, Brain natriuretic peptide, Electrocardiogram, ST change, Atrial fibrillation

Abbreviations: HD, Hemodialysis; CVD, Cardiovascular disease; Development dataset, Dataset for the development of risk score; Validation dataset, Dataset for validation of risk score; BMI, Body mass index; DM, Diabetes mellitus; ESRD, End-stage renal disease; CTR, Cardiothoracic ratio; ANP, Atrial natriuretic peptide; BNP, Brain natriuretic peptide; ECG, Electrocardiograms; In(vintage), Logarithm values of vintage; Cox PHMs, Cox proportional hazard models; HR, Hazard ratios; Cl, Confidence interval

\footnotetext{
*Correspondence: itoh@cepp.ne.jp

'Sakakibara Heart Institute, 2-4 Nishishinnjuku, Shinjuku-ku, Tokyo 163-0804,

Japan

Full list of author information is available at the end of the article
} 


\section{Background}

The number of patients on maintenance hemodialysis (HD) has been rapidly increasing over the last decades [1], and more than 320,000 patients are on maintenance HD in Japan at the end of 2014 [2]. This phenomenon has been attributed to the increase in the number of patients with diabetes (the main cause of renal failure in Japan) along with the advances in the techniques of HD and medical therapy for renal failure. However, the poor survival of dialysis patients has been reported by a number of investigators [3]. Cardiovascular disease (CVD) is common among these patients and is the primary cause of death in this population [4].

There were few papers that reported the impact of cardiac and/or circulatory disorder parameters, which are routinely measured along with vital signs during HD treatment, on long-term prognosis. To evaluate HD patients' prognosis and find patients with high risk of death, there is a need for novel index that is based on the cardiac disorder parameters. We, therefore, conducted a long-term prospective multicenter cohort study to establish a prediction model for mortality in maintenance HD patients using not specific parameters but clinically easy to measure parameters.

\section{Methods}

\section{Study design and study population}

This study conducted in two medical corporations operating seven HD clinics and one hospital in total and HD units of a general hospital in Japan as a multicenter observational study. The participating facilities were as follows: Tokiwa-kai Medical Corporation Group (Iwaki Urological Clinic, Izumi Clinic), Meysey-kai Medical Corporation Group (Airport Urological Clinic, Yokaichiba Clinic, Yachimata Clinic, Togane Clinic, Oami Neurosurgery Clinic, Mitsuhashi Hospital), and Chiba Social Insurance Hospital (Current name: JCHO Chiba Hospital). This study was approved by the ethics committees of all participating institutions (Meysey-kai Medical Corporation Group No. 215070001, Tokiwa-kai Medical Corporation Group No. 19-2, JCHO Chiba Hospital No. 45), and the research was conducted in accordance with the ethical principles of the Declaration of Helsinki. Informed consent was obtained by providing a document containing all the required elements of informed consent that gives patients the option to provide permission.

All the patients who received maintenance HD in these sites between October 1997 and April 1999 were enrolled (Fig. 1). Patients under 20 years, under the treatment of cancer, and on HD for less than 1 month were excluded. Patients with missing values and apparent outliers were also excluded $(n=102)$. The remaining patients were randomly classified into two groups to obtain (1) a dataset for the development of risk score (development dataset) and (2) a dataset for validation of risk score (validation dataset).

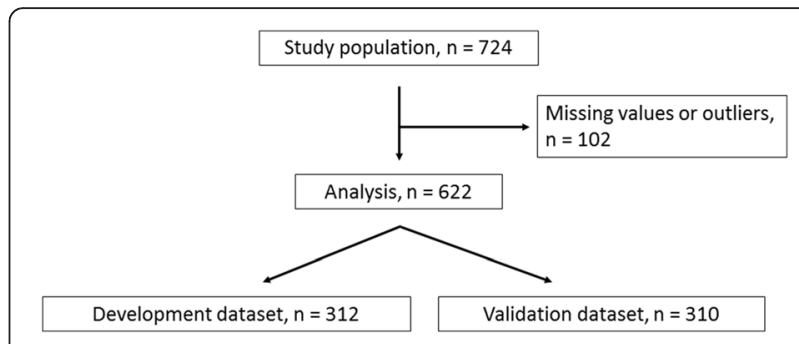

Fig. 1 Flow diagram of participants

\section{Data}

The patients' demographics, namely, age, gender, body mass index (BMI) calculated on the basis of post-HD body weight, diabetes mellitus (DM) as a cause of end-stage renal disease (ESRD), history of CVD, hypertension, hypotension during $\mathrm{HD}$, pre-HD cardiothoracic ratio (CTR, \%), pre-HD hemoglobin level (g/dl), pre-HD plasma atrial natriuretic peptide (ANP, pg/ml) level, brain natriuretic peptide (BNP, pg/ml) level, and findings on pre-HD electrocardiograms (ECGs) were obtained. CVD was defined as myocardial infarction, heart failure, arrhythmia, cerebral hemorrhage, and brain infarction. The primary endpoint was all-cause mortality, and the secondary was cardiovascular mortality. Hypertension was defined as a condition (1) requiring the use of the following types of antihypertensive drug: calcium channel blockers, angiotensin-converting enzyme inhibitors, angiotensin receptor blockers, and beta blockers or (2) having pre-HD hypertension (systolic blood pressure $160 \mathrm{mmHg}$ or higher, diastolic blood pressure $100 \mathrm{mmHg}$, or higher). Hypotension during HD was defined as a condition requiring the use of using the following medicines during HD: amezinium metilsulfate, droxidopa, etilefrine hydrochloride, midodrine hydrochloride, rapid infusion of normal saline, and an injection of $10 \%$ saline or $50 \%$ glucose solution. Abnormal findings on ECGs were (1) a horizontal ST segment depression of more than $1 \mathrm{~mm}$ or a negative $\mathrm{T}$ wave, (2) an abnormal Q wave, and (3) atrial fibrillation. The number of abnormal findings was used as the ECG score (0 to 3$)$.

\section{Follow-up}

Patients were prospectively followed up at the same clinics or hospitals. The data on mortality were examined by analyzing medical records from the outpatient clinic and conducting telephone interviews with the patients or their families. The data base was implemented in February 2014, with prospective data collection on all patients. At the end of December 2014, data were fixed and collected at the data center and analyzed independently of the participating investigator. 


\section{Statistical analyses}

Variables are expressed as mean \pm standard deviation. For variables not normally distributed, natural logarithm values were considered, i.e., the natural logarithm values of vintage $[\ln ($ vintage)]. Intergroup comparisons of parameters were performed using chi-square test, $t$ test, and Mann-Whitney $U$ test as appropriate. Age was scored into two categories (older): 0, less than 65 years; 1, 65 years or more. BMI was categorized into two values (low BMI): 1, less than $20.4 \mathrm{~kg} / \mathrm{m}^{2}$ (median); 0, $20.4 \mathrm{~kg} / \mathrm{m}^{2}$ or more. Controlled hemoglobin level was scored as 0 (10 to $12 \mathrm{~g} / \mathrm{dl}$ ) and 1 (other levels). Pre-HD CTR was categorized into two values (high CTR) based on the Japanese Society for Dialysis Therapy Guidelines for Management of Cardiovascular Diseases in Patients on Chronic Hemodialysis: 0, less than $50 \%$; 1, 50 \% or higher. Pre-HD plasma ANP level was categorized into two values (high ANP level): 0, less than $132.5 \mathrm{pg} / \mathrm{mL} ; 1$, $132.5 \mathrm{pg} / \mathrm{mL}$ or higher. Pre-HD plasma BNP level was categorized into two values (high BNP level): 0, less than $250 \mathrm{pg} / \mathrm{mL} ; 1,250 \mathrm{pg} / \mathrm{mL}$ or higher. The cutoff values of pre-HD plasma ANP and BNP levels were determined by sensitivity analysis. In statistical analysis, because the number of the patients with ECG score of 3 was small, patients with an ECG score $=3$ were treated as having ECG score $=2$. The primary outcome was all-cause death within 15 years. The other outcomes evaluated were as follows: all-cause death within 10 and 5 years and CVD-caused death within 15, 10, and 5 years.

Step 1: (1) Each candidate variable for a risk scoring model was selected from the development dataset using each of the Cox proportional hazard models (PHMs). Candidate Cox PHMs for the risk scoring model were constructed using the hierarchical backward elimination procedure. The initial multivariate Cox PHM was constructed including the selected variables. When variables were not statistically significant in the model, the variables were deleted and the next models were constructed until all variables were statistically significant $(p<0.05)$. (2) To develop a risk scoring model, we assigned each variable in the final model a weighted score proportional to the smallest parameter estimate, which was rounded to the nearest integer. For example, a point of a variable was the parameter estimate of the variable divided by the smallest parameter estimate in the final model. For each patient, the risk score was calculated using the risk scoring model as the sum of the points. (3) On the basis of the categorical criteria of the risk score, the patients were divided into four groups using Kaplan Meier survival curves (groups 1 to 4). Then, the survival curve of each group was evaluated on the basis of Kaplan Meier survival curves. Cox PHMs were used to compare the risk of the outcome between the groups. Cox PHMs were adjusted for the variables that were not included in the final model, such as gender, $\ln$ (vintage), controlled hemoglobin level, and high ANP level. The results are presented here as hazard ratios (HR) with $95 \%$ confidence interval (CI).

Step 2: The risk score was calculated for each patient using the validation dataset. On the basis of the categorical criteria of the risk score, patients were divided into four groups. Patients' survival curves were evaluated by Kaplan Meier analysis. The risk of the outcome was compared between the groups using Cox PHMs adjusted for gender, $\ln$ (vintage), controlled hemoglobin level, and high ANP level. These analyses were conducted using SAS version 9.4 (SAS, Inc., NC, USA). Statistical significance was defined as $p<0.05$.

\section{Results}

The study population consisted of 622 patients. The mean duration of dialysis of the patients was 5.0 \pm 6.0 years (range, 0.1 to 32.0 years). The primary etiology of renal disease in these patients was chronic glomerulonephritis in 255 patients (35.2\%), diabetic nephropathy in 197 (27.2\%), nephrosclerosis in 72 (9.9\%), IgA nephritis in 31 (4.2\%), polycystic renal disease in 24 (3.3\%), and other diseases in 145 (20.0\%). There were 217 patients (30.0\%) with DM at the time of study registration. At baseline, medical therapy included calcium antagonists in 411 patients (56.7\%), angiotensin-converting enzyme inhibitors and/or angiotensin-II receptor blockers in 129 (17.8\%), nitrates in 137 (18.9\%), digoxin in 48 (6.6\%), and beta blockers in 36 (4.9\%) in varying combinations.

After randomization, 312 patients were included for obtaining data for the development dataset and 310 patients for obtaining those for the validation dataset (Fig. 1). Their demographics including biochemical data are shown in Table 1. CTR, plasma BNP level, the number of the patients with high BNP level, and the numbers of all-cause death and CVD-caused death within 15 and 10 years in the validation dataset were higher than those in the development dataset.

\section{Development and categorization of risk score}

After the selection of the variables, the initial model was constructed. Ln(vintage) and controlled hemoglobin level were not included in the model: $\ln ($ vintage), $p=0.35$; controlled hemoglobin level, $p=0.43$. The initial model included the following variables: gender, older, DM, history of CVD, hypotension, high CTR, high ANP level, high BNP level, and ECG score. However, in the initial model, gender, low BMI, history of CVD, and high ANP level were not statistically significant: gender, $p=0.74$; low BMI, $p=0.25$; history of CVD, $p=0.69$; high ANP level, $p=0.99$. Then, the final model included older, DM, hypotension, high CTR, high BNP level, and ECG score. 
Table 1 Baseline characteristics

\begin{tabular}{|c|c|c|c|c|}
\hline & All & Development dataset & Validation dataset & $P$ value \\
\hline N & 622 & 312 & 310 & \\
\hline Male (\%) & $376(60.45)$ & $191(61.22)$ & $185(59.68)$ & 0.69 \\
\hline Age (years) & $52.9 \pm 14.1$ & $51.9 \pm 14.3$ & $53.9 \pm 14.0$ & 0.07 \\
\hline Older (\%) & $133(22.09)$ & 63 (20.19) & $70(22.58)$ & 0.47 \\
\hline \multirow[t]{2}{*}{ Vintage (years) } & $5.7 \pm 6.0$ & $5.6 \pm 5.7$ & $5.9 \pm 6.3$ & \multirow[t]{2}{*}{0.84} \\
\hline & $3.4(1.2,8.0)$ & $3.5(1.2,7.8)$ & $3.3(1.3,8.3)$ & \\
\hline BMI $\left(\mathrm{kg} / \mathrm{m}^{2}\right)$ & $20.7 \pm 2.7$ & $20.6 \pm 2.7$ & $20.7 \pm 2.8$ & 0.83 \\
\hline Low BMI (\%) & $290(46.62)$ & $146(46.79)$ & $144(46.45)$ & 0.93 \\
\hline DM (\%) & $170(27.33)$ & $78(25)$ & $92(29.68)$ & 0.19 \\
\hline History of CVD & $72(11.58)$ & $35(11.22)$ & 37 (11.94) & 0.78 \\
\hline Hypertension & $619(99.52)$ & 310 (99.36) & 309 (99.68) & 0.57 \\
\hline Pre-HD hypertension & 619 (99.52) & 310 (99.36) & 309 (99.68) & 0.57 \\
\hline Antihypertensive drug use & $378(60.77)$ & $196(62.82)$ & $182(58.71)$ & 0.29 \\
\hline Hypotension during HD & $218(35.05)$ & 105 (33.65) & $113(36.45)$ & 0.46 \\
\hline CTR (\%) & $50.7 \pm 5.0$ & $50.2 \pm 5.1$ & $51.2 \pm 5.0$ & 0.02 \\
\hline High CTR (\%) & $330(53.05)$ & $155(49.68)$ & $175(56.45)$ & 0.09 \\
\hline Hemoglobin level (g/dL) & $9.5 \pm 1.1$ & $9.4 \pm 1.0$ & $9.6 \pm 1.2$ & 0.07 \\
\hline Controlled hemoglobin level (\%) & 189 (30.39) & $87(27.88)$ & $102(32.9)$ & 0.17 \\
\hline \multirow[t]{2}{*}{ Plasma ANP level (pg/mL) } & $182.4 \pm 153.2$ & $180.0 \pm 170.8$ & $184.8 \pm 133.5$ & \multirow[t]{2}{*}{0.10} \\
\hline & $143(87.4,238)$ & $132.5(79.9,230.0)$ & $149.5(91.6,241.0)$ & \\
\hline High ANP level (\%) & $472(75.88)$ & $231(74.04)$ & $241(77.74)$ & 0.28 \\
\hline \multirow[t]{2}{*}{ Plasma BNP level (pg/mL) } & $474.4 \pm 613.4$ & $470.2 \pm 699.3$ & $478.6 \pm 513.7$ & \multirow[t]{2}{*}{0.04} \\
\hline & $291.5(150,587)$ & $261.5(131.0,508.0)$ & $335.0(166.0,614.0)$ & \\
\hline High BNP level (\%) & $477(76.69)$ & $227(72.76)$ & $250(80.65)$ & 0.02 \\
\hline ST segment depression or negative T wave (\%) & $221(35.53)$ & $108(34.62)$ & $113(36.45)$ & 0.63 \\
\hline Abnormal Q wave (\%) & $39(6.27)$ & $20(6.41)$ & $19(6.13)$ & 0.89 \\
\hline Atrial fibrillation (\%) & $14(2.25)$ & $4(1.28)$ & $10(3.23)$ & 0.10 \\
\hline \multicolumn{3}{|l|}{ ECG score (\%) } & & \multirow[t]{5}{*}{0.59} \\
\hline 0 & $380(61.09)$ & $195(62.5)$ & $185(59.68)$ & \\
\hline 1 & $211(33.92)$ & $103(33.01)$ & $108(34.84)$ & \\
\hline 2 & $30(4.82)$ & $13(4.17)$ & $17(5.48)$ & \\
\hline 3 & $1(0.16)$ & $1(0.32)$ & $0(0)$ & \\
\hline All-cause death within 15 years (\%) & $409(65.76)$ & $200(64.1)$ & $209(73.87)$ & 0.01 \\
\hline All-cause death within 10 years (\%) & $342(54.98)$ & $155(49.68)$ & $187(60.32)$ & 0.01 \\
\hline All-cause death within 5 years (\%) & $197(31.67)$ & $96(30.77)$ & $101(32.58)$ & 0.63 \\
\hline CVD-caused death within 15 years (\%) & $230(36.98)$ & $102(32.69)$ & $128(55.65)$ & 0.03 \\
\hline CVD-caused death within 10 years (\%) & $197(31.67)$ & $86(27.56)$ & $111(35.81)$ & 0.03 \\
\hline CVD-caused death within 5 years (\%) & $117(18.81)$ & $56(17.95)$ & $59(19.03)$ & 0.73 \\
\hline \multirow[t]{2}{*}{ Follow-up period (days) } & $2963.4 \pm 1839.3$ & $3043.7 \pm 1904.8$ & $2882.5 \pm 1770.5$ & \multirow[t]{2}{*}{0.28} \\
\hline & $2813(1301,5040)$ & $2908(1246,5182)$ & $2712(1319,4584)$ & \\
\hline
\end{tabular}

Variables are expressed as mean \pm standard deviation. Vintage, plasma ANP and BNP levels, and follow-up days are also shown as median and interquartile range. Intergroup comparisons of parameters were performed using chi-square test, $t$ test, and Mann Whitney $U$ test as appropriate as appropriate

Development dataset dataset for the development of risk score, Validation dataset dataset for the validation of risk score, Older $65 \leq$ age, BMI body mass index, Low $B M I \mathrm{BMI}<20.4 \mathrm{~kg} / \mathrm{m}^{2}$, DM diabetes mellitus as a cause of end-stage renal disease, CVD cardiovascular disease, HD hemodialysis, CTR cardiothoracic ratio, High CTR $50 \% \leq \mathrm{CTR}, A N P$ atrial natriuretic peptide, High ANP level $132.5 \mathrm{pg} / \mathrm{mL} \leq \mathrm{plasma}$ ANP level, BNP brain natriuretic peptide, High BNP level $250 \mathrm{pg} / \mathrm{mL} \leq \mathrm{plasma}$ BNP level, ECG score the number of abnormal findings in electrocardiogram 
The risk scoring model was developed using the parameter estimates in the final model as follows (Table 2):

$$
\begin{aligned}
\text { Risk score }= & \text { older }+ \text { DM }+ \text { hypotension } \\
& + \text { high CTR }+ \text { high BNP level } \\
& + \text { ECG score }
\end{aligned}
$$

Older, yes $=3$, no $=0 ; \mathrm{DM}$, yes $=3$, no $=0$; hypotension, yes $=1$, no $=0$; high CTR, yes $=1$, no $=0$; high BNP level, yes $=1$, no $=0$; ECG score, score $0=0$, score $1=1$, score $2=5$.

The Kaplan Meier survival curves showed a significant difference in the survival probability of the patients determined on the basis of risk score (log-rank test $p=$ 0.0001). The patients were categorized into four groups on the basis of the risk score: group 1 (low risk), risk score $=0$; group 2, score $=1$ to 3 ; group 3 , score $=4$ to 5 ; and group 4 (high risk), score $=6$ and higher. The Kaplan Meier survival curve showed the difference in the survival probability of the groups (log-rank test $p=0.0001$ ) (Fig. 2). Groups 2 to 4 showed higher risks of all-cause death than group 1: group 2, HR $4.2995 \%$ CI $(2.29,8.03)$, adjusted HR 4.1995 \% CI (2.22, 7.91); group 3, HR 14.4795 \% CI (7.55, 27.72), adjusted HR $14.6895 \%$ CI $(7.58,28.41)$; group 4, HR 21.84 95 \% CI $(10.96,43.53)$, adjusted HR $24.2995 \%$ CI $(11.83,49.89)$.

\section{Validation of risk score and its categories}

Using the validation dataset, we compared the risk of death between the groups. The risks of all-cause death and CVD-caused death in group 4 were higher than those in other groups (Table 3). The Kaplan Meier survival curves for all-cause death and CVD-caused death showed a lower survival probability in group 4 than in the other groups for 15 years (Fig. 3). Moreover, groups 2 to 4 showed high risks of all-cause death and CVD-caused death than group 1 (Table 4).

Table 2 Parameter estimates in the final models and risk score

\begin{tabular}{llll}
\hline Variables & Parameter estimate in the final model & Ratio & Score \\
\hline Older & 0.96 & 2.97 & 3 \\
DM & 0.85 & 2.63 & 3 \\
Hypotension & 0.41 & 1.25 & 1 \\
High CTR & 0.38 & 1.16 & 1 \\
High BNP level & 0.32 & 1 & 1 \\
ECG score $=1$ & 0.46 & 1.43 & 1 \\
ECG score $=2$ & 1.52 & 4.69 & 5 \\
\hline
\end{tabular}

Each parameter estimate in the final models was compared with the smallest parameter estimate (High BNP level). Then, the risk scores were determined. Older $65 \leq$ age, DM diabetes mellitus as a cause of end-stage renal disease, Hypotension hypotension during hemodialysis, High CTR $50 \% \leq$ cardiothoracic ratio, High BNP level $250 \mathrm{pg} / \mathrm{mL} \leq$ plasma brain natriuretic peptide level, ECG score the number of abnormal findings in electrocardiogram

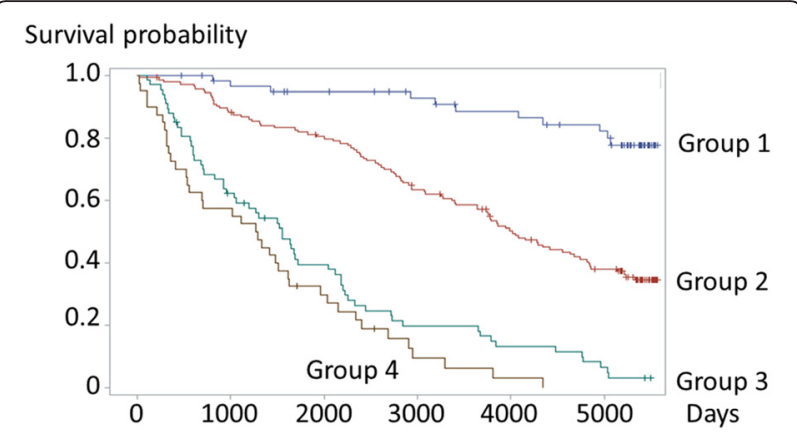

Fig. 2 Association between groups and mortality in development dataset. Kaplan Meier survival curves showed that group 1 had the highest survival probability

\section{Discussion}

Although the prognosis of the HD patients in Japan is relatively good compared with that in other countries, the leading cause of death is also cardiovascular disease [2]. Several reports have been published that disclosed the usefulness of particular factors related to cardiovascular disorder for predicting a HD patient's prognosis. Such factors are coronary artery disease [5], hypertension [6], left ventricular hypertrophy [7], hypotension [8], left ventricular function [9], left ventricular size [10], BNP level [11], atrial fibrillation [12], hypocholesterolemia [13], cardiac troponin [14], C-reactive protein level [15], and autonomic nervous system abnormality [16] among others. However, it has never been considered the contribution rate of each factor for the mortality of patients on maintenance HD. Moreover, there was no report on the scoring system for predicting the long-term prognosis in those patients, considering that the vintage of dialysis patients has been increasing with a quarter of them receiving HD more than 10 years in Japan [2]. In this study, we proposed a scoring system using routine measurements to predict the long-term prognosis of HD patients.

We evaluated the patients' basic information, such as age, gender, physical constitution, DM as a cause of ESRD, and history of CVD, which appears in the very first page of the patients' chart. Chest X-ray images and ECGs are obtained routinely, and hemoglobin and natriuretic peptide levels are also frequently measured at the beginning of a regular HD session. Blood pressure is routinely measured during HD to ensure safe treatment. In this study, we focused on these common parameters that are obtained routinely at the beginning of maintenance HD and during maintenance HD treatment.

As a result, most of the significant parameters listed in this study were found to be related to cardiac and/or circulatory disorders. DM is a strong risk factor for atherosclerosis, and abnormal Q wave and ST depression are signs of coronary artery disease. High BNP level, high CTR, and hypotension during HD treatment are mainly 
Table 3 Risks of death in groups on the basis of risk scores in validation dataset

\begin{tabular}{llll}
\hline Follow-up period & 15 years & 10 years & 5 years \\
\hline All-cause death & & & $0.039(0.001,0.091)$ \\
Group 1 & $0.15(0.06,0.25)$ & $0.14(0.042,0.23)$ & $0.23(0.16,0.30)$ \\
Group 2 & $0.58(0.50,0.67)$ & $0.42(0.34,0.51)$ & $0.49(0.36,0.61)$ \\
Group 3 & $0.92(0.86,0.99)$ & $0.74(0.64,0.85)$ & $0.65(0.50,0.80)$ \\
Group 4 & $0.98(0.93,0.99)$ & $0.93(0.84,0.99)$ & $0.019(0.001,0.057)$ \\
CVD-caused death & & & $0.088(0.040,0.14)$ \\
Group 1 & $0.12(0.029,0.20)$ & $0.21(0.14,0.28)$ & $0.32(0.21,0.43)$ \\
Group 2 & $0.26(0.18,0.33)$ & $0.50(0.38,0.62)$ & $0.40(0.25,0.55)$ \\
Group 3 & $0.55(0.43,0.67)$ & $0.58(0.42,0.73)$ & $0.18)$ \\
Group 4 & $0.60(0.45,0.75)$ & & $0.096)$ \\
\hline
\end{tabular}

Values are risks of death with $95 \%$ confidence intervals in each group. Patients were categorized into four groups on the basis of their risk scores CVD cardiovascular disease

due to impaired cardiac function and disorder of hemodynamics. Shoji et al. reported that hypotension during HD treatment, which is a significant prognostic parameter, is closely related to interdialysis body weight gain beside age and vintage [8]. This condition is considered to suggest a chronic volume overload and an acute change in loading condition caused by HD treatment.

Heart failure, DM, and aging are also risk factors for arrhythmias. Regarding the cardiac arrhythmia in HD patients, ventricular arrhythmia was focused on as a possible to cause cardiac death [17]. Although the incidence and severity of ventricular premature beats were high, there was no direct evidence that ventricular arrhythmia itself is related to the prognosis in these patients. It has been documented that atrial fibrillation affects the prognosis in non-ESRD patients [18] and its prevalence increases with aging [19]. Atrial fibrillation is much more frequent in HD patients than in the general population; age, HD vintage, presence of some heart diseases, and left atrial dilatation are associated with the arrhythmia [20]. Va'zquez et al. reported that atrial fibrillation itself worsen the prognosis of HD patients [12].
We developed a scoring system for predicting the long-term prognosis of chronic HD patient using these parameters. In Japan, the same set of examinations is commonly performed in all the HD patients routinely in many facilities. This scoring system provides us proper usage of medical resources in the medical care of ESRD. The scoring system has several features. First, the scoring system we developed in this study includes markers commonly used to evaluate cardiac disease, which were easily measurable and did not require special skills of examiners. Moreover, the scoring system can be used to evaluate HD patients' long-term prognosis. Previous scores were developed to evaluate HD patients' short-term mortality $[21,22]$. These scores can be used separately. For examination, the scoring system of this study is used to evaluate a HD patient's long-term prognosis. If the patient has a high risk of death, his prognosis will be evaluated using a score for short-term prognosis, which can exactly predict his prognosis. HD patients' prognosis is determined by many factors. The scores were differently developed on the basis of the population and purpose. Because a single score cannot cover all of the patients with many

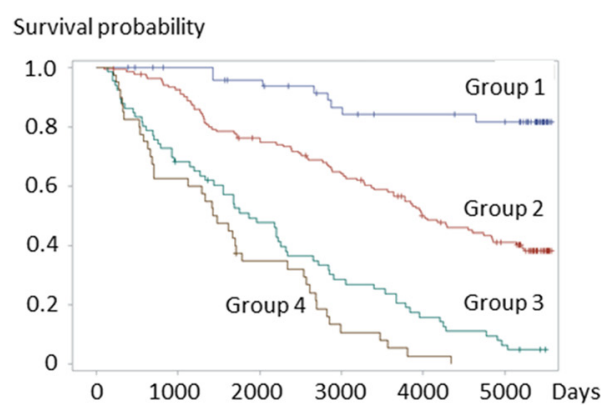

A All-cause death

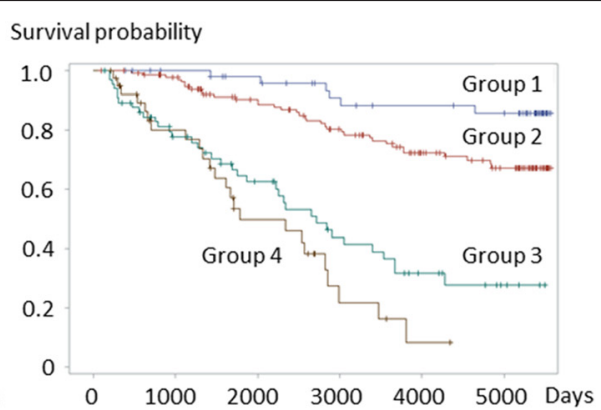

B CVD-caused death

Fig. 3 Association between groups and mortality in validation dataset. Kaplan Meier survival curves showed that group 1 had the highest survival probability free from all-cause death (a) and CVD-caused death (b). CVD cardiovascular disease 
Table 4 Groups with high risk scores and high risks of death

\begin{tabular}{cllllll}
\hline Follow-up period & 15 years & \multicolumn{3}{l}{5 years } \\
\hline All-cause death & HR $(95 \% \mathrm{Cl})$ & aHR $(95 \% \mathrm{Cl})$ & $\mathrm{HR}(95 \% \mathrm{Cl})$ & aHR $(95 \% \mathrm{Cl})$ & $\mathrm{HR}(95 \% \mathrm{Cl})$ & aHR $(95 \% \mathrm{Cl})$ \\
Group 1 & Reference & Reference & Reference & Reference & Reference & Reference \\
Group 2 & $4.66(2.25,9.64)$ & $4.56(2.20,9.49)$ & $3.48(1.59,7.62)$ & $3.23(1.47,7.10)$ & $6.43(1.54,26.80)$ & $5.71(1.36,23.92)$ \\
Group 3 & $13.62(6.48,28.63)$ & $15.23(7.17,32.34)$ & $9.41(4.25,20.80$ & $10.06(4.50,22.48)$ & $16.89(4.05,70.44)$ & $17.29(4.11,72.65)$ \\
Group 4 & $20.86(9.60,45.31)$ & $24.96(11.21,55.59)$ & $14.796 .56,33.34)$ & $17.02(7.36,39.33)$ & $24.761(5.88,104.34)$ & $25.25(5.89,108.23)$ \\
CVD-caused death & HR $(95 \% \mathrm{Cl})$ & aHR $(95 \% \mathrm{Cl})$ & $\mathrm{HR}(95 \% \mathrm{Cl})$ & aHR $(95 \% \mathrm{Cl})$ & $\mathrm{HR}(95 \% \mathrm{Cl})$ & aHR $(95 \% \mathrm{Cl})$ \\
Group 1 & Reference & Reference & Reference & Reference & Reference & Reference \\
Group 2 & $2.59(1.09,6.17)$ & $2.51(1.05,6.01)$ & $2.45(0.95,6.34)$ & $2.32(0.89,6.03)$ & $4.84(0.63,37.21)$ & $4.61(0.60,35.58)$ \\
Group 3 & $9.62(4.03,22.96)$ & $10.79(4.45,26.17)$ & $9.20(3.58,23.62)$ & $10.17(3.90,26.49)$ & $22.26(2.99,165.50)$ & $25.89(3.45,194.30)$ \\
Group 4 & $14.78(5.95,36.74)$ & $19.06(7.36,49.31)$ & $13.70(5.17,36.32)$ & $17.27(6.27,47.54)$ & $30.66(4.06,231.34)$ & $37.71(4.87,291.93)$ \\
\hline
\end{tabular}

Values are HRs with $95 \% \mathrm{Cls}$ of all groups compared with group 1. Groups 2 to 4 show higher risks of death than group 1. Patients were categorized into four groups on the basis of their risk scores

CVD cardiovascular disease, $H R$ hazard ratio, $a H R$ adjusted hazard ratio, $\mathrm{Cl}$ confidence interval

conditions, we should select properly a score on the basis of a patient's condition.

This study has several limitations. First, we were unable to examine the patients with missing data in this study, which might have caused selection bias. Second, the dataset did not include sufficient data for assessing nutrition, chronic kidney disease-mineral and bone disorder, comorbid conditions, and medications. Clinical practice guidelines for the management in hemodialysis patients established by the Japanese Society for Dialysis Therapy have been implemented since 2004. Although the markers in this study were selected in 1997, they are still commonly used. Third, although we developed and validated the risk scoring system using the different datasets, the population of the present study cannot be said to represent the HD patients in Japan. And there were differences in the characteristics of the development dataset and validation datasets such as the numbers of all-cause death and CVD-caused death. The differences might cause bias for the risk score. However, the validation of the scoring system showed a possibility that the scoring system was applicable to a population with different risk. Further validation study is required. Fourth, we used dichotomized variables. Although this strategy simplifies the development of a risk scoring system, the use of continuous variables may provide more refined information [21]. Fifth, this study was carried out from 1997 to 2014. During this period, various new medicines were developed such as angiotensin II receptor blockers and erythropoiesis-stimulating agents. Moreover, various guidelines were established by the Japanese Society for Dialysis Therapy. We were unable to time-dependently evaluate the effects of these innovations on the prognosis of the subjects. This might have made bias on this scoring system, however, because the scoring system did not include medications and all of the subjects had equal opportunity to benefit from these innovations. The effects of bias might be reduced, and the categorization of the subjects from high-risk group to low-risk group may have minimized errors.

\section{Conclusions}

In conclusion, this study shows the significant association between long-term prognosis and cardiovascular diseaserelated risk factors in HD patients and we developed and validated a new simple scoring system for predicting their prognosis spanning long periods of time. This will also contribute not only to the grading of the risk of HD patients but also to deliver medical resources to the right patients.

\section{Acknowledgements \\ None. \\ Funding \\ There was no funding.}

Availability of data and materials

The data will not be shared because the informed consent from the subjects regarding the public availability for their personal data was not obtained.

\section{Authors' contributions}

$\mathrm{HI}$ (Haruki Itoh) carried out the planned and performed the whole study, acquired the data, performed analysis, and created the manuscript. HK, YT, NM, TM, and HI (Hidetaka) designed the study, acquired the data, performed the interpretation of the data, and revised the manuscript. EK performed analysis and interpretation of the data and revised the manuscript. All authors read and approved the final manuscript.

\section{Competing interests}

The authors declare that they have no competing interests.

\section{Ethics approval and consent to participate}

All procedures performed in studies involving human participants were in accordance with the ethical standards of the institutional research committee at which the studies were conducted (IRB approval number 215070001, 19-2, 45) and with the Helsinki declaration and its later amendments or comparable ethical standards. And informed consent was obtained from all individual participants included in the study. 


\section{Author details}

'Sakakibara Heart Institute, 2-4 Nishishinnjuku, Shinjuku-ku, Tokyo 163-0804, Japan. ${ }^{2}$ Tokiwa-kai Medical Corporation, Iwaki-shi, Fukushima-ken, Japan. ${ }^{3}$ Meysey-kai Medical Corporation, Togane-shi, Chiba-ken, Japan.

${ }^{4}$ Hemodialysis Department, Japan Community Health Care, Organization Chiba Hospital, Chiba-shi, Chiba-ken, Japan. ${ }^{5}$ Clinical Examination

Department, Sakakibara Heart Institute Clinic, Shinjuku-ku, Tokyo, Japan.

${ }^{6}$ Department of Internal Medicine, Toranomon Mutual Aid General Hospital,

Minako-ku, Tokyo, Japan. ${ }^{7}$ Department of Nephrology, Tokyo Kyosai Hospital,

Meguro-ku, Tokyo, Japan.

Received: 25 June 2016 Accepted: 25 August 2016

Published online: 13 October 2016

\section{References}

1. Pastan S, Bailey J. Dialysis therapy. N Engl J Med. 1998;338:1428-37.

2. Masakane I, Nakai S, Ogata S, Kimata N, Hanabusa N, Hamano T, Wakai T, Wada A, Nitta K. An overview of regular diadysis treatment in Japan (as of December 31, 2014). J Jpn Soc Dial Ther. 2016;49:1-34.

3. United States renal deta system. http://www.usrds.org/2014/view/Default.aspx. Accessed 31 Aug 2016.

4. Lindner A, Charra B, Sherrard DJ. Accelerated atherosclerosis in prolonged maintenance hemodialysis. N Engl J Med. 1974;290:697-701.

5. Herzog CA, Ma LZ, Collins AJ. Poor long-term survival after acute myocardial infarction among patients on long-term hemodialisys. N Eng J Med. 1998:339:799-805

6. Bansal N, McCulloch CE, Rahman M, Kusek JW, Anderson AH, Xie D, Townsend RR, Lora CM, Wright J, Go AS, Ojo A, Alper A, Lustigova E, Cuevas M, Kallem R, Hsu C, the CRIC Study Investigators. Blood pressure and risk of all-cause mortality in advanced chronic kidney disease and hemodialysis. The chronic renal insufficiency cohort study. Hypertension. 2015;65:93-100.

7. Lopez-Gomez JM, Verde E, Perez-Garcia R. Blood pressure, left ventricular hypertrophy and long-term prognosis in hemodialysis patients. Kidney Int. 1998:68:S92-8

8. Shoji T, Tsubakihara Y, Fujii M, Imai E. Hemodialysis-associated hypotension as an independent risk factor for two-year mortality in hemodialysis patients. Kidney Int. 2004:66:1212-20.

9. Yamada S, Ishii H, Takahashi H, Aoyama T, Morita Y, Kasuga H, Kimura K, Ito Y, Takahashi R, Toriyama T, Yasuda Y, Hayashi M, Kamiya H, Yuzawa Y, Maruyama S, Matsuo S, Matsubara T, Murohara T. Prognostic value of reduced left ventricular ejection fraction at start of hemodialysis therapy on cardiovascular and all-cause mortality in end-stage renal disease patients. Clin J Am Soc Ncphrol. 2010;5:1793-8.

10. Inoue T, Ogawa T, Iwabuchi Y, Otsuka K, Nitta K. Left ventricular end-diastolic diameter is an independent predictor of mortality in hemodialysis patients? Ther Aphresis Dial. 2012;16:134-41.

11. Naganuma T, Sugimura K, Wada S, Yasumoto R, Sugimura T, Masuda C, Uchida J, Nakatani T. The prognostic role of brain natriuretic peptides in hemodialysis patients. Am J Nephrol. 2002;22:437-44.

12. Va'zquez E, Sa'nchez-Perales C, Lozano C, Garci'a-Corte's MJ, Borrego F, Guzma'n M, Pe'rez P, Pagola C, Borrego MJ, Pe'rez V. Comparison of prognostic value of atrial fibrillation versus sinus rhythm in patients on long-term hemodialysis. Am J Cardiol. 2003;92:868-71.

13. Iseki K, Yamazato M, Tozawa M, Takishita S. Hypocholesterolemia is a significant predictor of death in a cohort of chronic hemodialysis patients. Kidney Int. 2002;61:1887-93.

14. Khan NA, Hcmmelgarn BR, Tonelli M, Thompson CR, Levin A. Prognostic value of troponin $T$ and I among symptomatic patients with end-stage renal disease: a meta-analysis. Circulation. 2005;112:3088-96.

15. Yeun J, Levine R, Mantadilok V, Kaysen G. C-reactive protein predicts all-cause and cardiovascular mortality in hemodialysis patients. Am J Kidney Dis. 2000;35:469-76

16. Oikawa K, Ishihara R, Maeda T, Yamaguchi K, Koike A, Kawaguchi H, Tabata Y, Murotani $\mathrm{N}$, Itoh H. Prognostic value of heart rate variability in patients with renal failure on hemodialysis. Int J Cardiol. 2009;131:370-7.

17. Morrison G, Michelson E, Brown S, Morganroth J. Mechanism and prevension of cardiac srrhythmias in chronic hemodialysis patioents. Kidney Int. 1980;17:811-9.

18. The AFFIRM Investigators. Relationship between sinus rhythm, treatment, and survival in the atrial fibrillation follow-up investigation of rhythm management (AFFIRM) study. Circulation. 2004;109:1509-13.
19. Kannel WB, Wolf PA, Benjamin EJ, Levy D. Prevalence, incidence, prognosis, and predisposing conditions for atrial fibrillation: population-based estimates. Am J Cardiol. 1998;82:2N-9.

20. Genovesi S, Pogliani D, Faini A, Valsecchi MG, Riva A, Stefani F, Acquistapace I, Stella A, Bonforte G, DeVecchi A, DeCristofaro V, Buccianti G, Vincenti A. Prevalence of atrial fibrillation and associated factors in a population of long-term hemodialysis patients. Am J Kidney Dis. 2005;46:897-902.

21. Kanda E, Bieber BA, Pisoni RL, Robinson BM, Fuller DS. Importance of simultaneous evaluation of multiple risk factors for hemodialysis patients' mortality and development of a novel index: dialysis outcomes and practice patterns study. PLoS One. 2015;10:e0128652.

22. Anker SD, Gillespie IA, Eckardt KU, Kronenberg F, Richards S, Drueke TB, Stenvinkel P, Pisoni RL, Robinson BM, Marcelli D, Froissart M, Floege J, On behalf the ARO Steering Committee (collaborators). Development and validation of cardiovascular risk scores for haemodialysis patients. Int J Cardiol. 2016;216:68-77.

\section{Submit your next manuscript to BioMed Central and we will help you at every step:}

- We accept pre-submission inquiries

- Our selector tool helps you to find the most relevant journal

- We provide round the clock customer support

- Convenient online submission

- Thorough peer review

- Inclusion in PubMed and all major indexing services

- Maximum visibility for your research

Submit your manuscript at www.biomedcentral.com/submit
) Biomed Central 